\title{
Pensamiento complejo, ética planetaria y transformación social en la formación del profesorados
}

\author{
Juan Martín López-Calva \\ ${ }^{1}$ http://orcid.org/0000-0002-6948-8556, Universidad Popular Autónoma del Estado de Puebla, México
}

Citar como: López-Calva, J. (2021). Pensamiento complejo, ética planetaria y transformación social en la formación del profesorados. Revista Digital de Investigación en Docencia Universitaria, 16(1), e1568. https://doi.org/10.19083/ridu.2022.1568

Recibido: 03/11/21. Revisado: 07/12/21. Aceptado: 08/02/22. Publicado: 25/02/22.

\begin{abstract}
Resumen
Introducción: La crisis que vive el mundo actual demanda la atención a la formación ética de las nuevas generaciones. Un elemento indispensable para atenderla es la formación ética de los docentes. Objetivo: Presentar los resultados de una investigación exploratoria que analizó la opinión de profesores y expertos en programas de capacitación docente sobre la formación ética del profesorado desde el bucle: pensamiento complejo-ética planetaria-transformación social. Método: Se encuestó a un grupo de 22 expertos en formación del profesorado y a 222 docentes de distintos niveles educativos, se les aplicó un cuestionario exploratorio sobre la formación ética del profesorado, construido con una escala Likert de siete opciones. Se presentan descriptiva y comparativamente los resultados de las preguntas relativas al bucle descrito en el objetivo, que es parte de la propuesta teórica construida. Resultados: muestran que no hay suficiente atención a la formación ética del profesorado desde las dimensiones: pensamiento complejo-ética planetaria- transformación social. Discusión: la opinión de los profesores es ligeramente más positiva que la de los expertos, aunque ambos grupos muestran la insuficiencia de la formación de los docentes en estos rubros. Al ser un estudio exploratorio, aporta una primera mirada comparativa. Será necesario complementarlo con un estudio cualitativo.
\end{abstract}

Palabras clave: pensamiento; responsabilidad del docente; cambio social; formación de docentes.

\section{Complex Thinking, Planetary Ethics and Social Transformation in Teacher Education}

\begin{abstract}
Introduction: The crisis that the world is experiencing today is demanding attention to the ethical training of the new generations. An essential element to attend it is the ethical training of teachers. Objective: To present the results of an exploratory research that investigated the opinion of teachers and experts in teacher training programs, on the ethical training of teachers from the loop: complex thinking-planetary ethics-social transformation. Method: A sample of 22 experts in teacher training and 222 teachers of different educational levels were surveyed, applying an exploratory questionnaire on the ethical training of teachers, constructed with a Likert scale of seven options. The results of the questions related to the loop described in the objective, which is part of the constructed theoretical proposal, are presented descriptively and comparatively. Results: show that there is not enough attention to the ethical training of teachers from
\end{abstract}


the dimensions: complex thought-planetary ethics-social transformation. Discussion: the opinion of the teachers is slightly more positive than that of the experts, although both groups show the insufficiency of teacher training in these areas. Being an exploratory study, it provides a first comparative look. It will be necessary to complement it with a qualitative study.

Keywords: thinking; teacher responsibility; social change; teacher education.

\section{Introducción}

La pandemia mundial por la COVID-19, enfermedad infecciosa causada por el virus SARS-CoV-2, que surgió en la ciudad de Wuhan, China, a fines del 2019 y se expandió rápidamente por el mundo durante los primeros meses del 2020, ha sido un llamado de atención urgente a la humanidad que necesita una transformación si quiere sobrevivir a los riesgos que implica el cambio climático y la destrucción sistemática de la naturaleza, que se ha venido incrementando desde el origen de la modernidad, a pesar de su promesa de progreso sin retorno.

Pero, lejos de cumplirse esta promesa, la pandemia ha amplificado los enormes problemas estructurales que han señalado muchos pensadores contemporáneos (Morin, 1993, 2020; Gore, 2006; Gorostiaga, 2000; Touraine, 1994, Wallerstein, 2007; entre otros) definiéndolos con diversos conceptos como crisis del sistema mundo, crisis estructural del capitalismo, crisis de la modernidad, era de hierro planetaria o cambio de época.

Como afirma Morin (2020), el mundo de hoy enfrenta enormes desafíos que se expresan en una crisis política y económica que parece anunciar una crisis alimentaria mundial, además de una crisis social de imprevisibles proporciones, que se está manifestando en grandes movimientos de migrantes que intentan llegar desde los países pobres a las naciones con economías fuertes. Todos estos desafíos son interdependientes, por lo que se tienen que abordar de forma sistémica.

En síntesis, se vive hoy una crisis de humanidad que es en el fondo una crisis ética, puesto que la ética estudia el proceso permanente, histórico y sociocultural de construcción del bien humano en sus niveles particular, estructural-social y de valor o cultural (Lonergan, 1998).

A esta crisis ética busca responder la agenda 2030 de la Organización de las Naciones Unidas (ONU), expresada en los Objetivos de desarrollo sostenible (ODS) (ONU, 2015) que fueron evaluados recientemente en su avance (ONU, 2020). El objetivo directamente relacionado con la educación es el número 4 que habla de: "Garantizar una educación inclusiva, equitativa y de calidad y promover oportunidades de aprendizaje durante toda la vida para todos ${ }^{1 "}$ que tiene en su redacción un claro posicionamiento ético, pero prácticamente todos los ODS están buscando responder a la crisis de religación humana (Morin, 2005), a la crisis ética que vive el mundo en las primeras décadas del siglo $\mathrm{XXI}^{2}$.

Una de las condiciones indispensables para responder al desafío ético que está planteando a la humanidad el cambio de época y que se manifiesta en prácticamente todos los campos del quehacer humano, es la formación ética de los profesores, puesto que ellos son responsables de la formación integral de los ciudadanos del futuro, que incluye como una dimensión fundamental la educación ética de los futuros ciudadanos.

De la relevancia de esta formación ética de los docentes de todos los niveles se han ocupado múltiples investigaciones entre las que se pueden citar las de Hirsch (2009; 2013; 2021), Pérez-Castro y Piña (2021), Belavi y Murillo (2020) y Meirieu (2022). En cuanto a los aspectos metodológicos de estos y otros trabajos sobre el tema, encontramos un buen número que utilizan cuestionarios con escalas Likert de opinión para conocer los valores y las conductas no éticas que se plantean los profesores y estudiantes como relevantes y presentes en la práctica docente. En cuanto a los enfoques teóricos, predominan en estos trabajos, en los ejemplos sistematizados por Hirsch (2009) dentro del Proyecto interuniversitario sobre Éti-

1 Cfr. https://www.un.org/sustainabledevelopment/es/ education/

2 Cfr. https://www.un.org/sustainabledevelopment/es/ 
ca profesional y en muchos otros trabajos sobre la formación ética del profesorado (Jover \& Ruiz, 2013; Ramos \& López, 2019), los sustentados en la ética de principios que no asume de entrada la incertidumbre y la complejidad de los fenómenos éticos, ni la relación entre pensamiento, ética y transformación social.

En este contexto, este trabajo constituye una aportación teórica novedosa sobre la formación ética del profesorado basada en el bucle: pensamiento complejo-ética planetaria- transformación social y hace un ejercicio de indagación empírica exploratoria metodológicamente similar a la de otros trabajos realizados en el tema de la ética del profesorado, como una primera aproximación empírica de este enfoque teórico basado en el paradigma de la complejidad (Morin, 2006) y en la propuesta filosófica humanista del filósofo canadiense Bernard Lonergan (1999).

El objetivo de este artículo es presentar los resultados de esta investigación exploratoria que indagó la opinión de profesores y expertos en programas de formación docente, sobre la formación ética del profesorado desde el bucle: pensamiento complejo-ética planetaria-transformación social.

\section{Sustento Teórico.}

La propuesta teórica original en la que se sustenta este artículo tiene como base la articulación de tres elementos que se consideran fundamentales para la buena formación ética de los profesores. Se trata de la unión dialógica entre el pensamiento complejo, la ética planetaria y la transformación social, que es parte de una investigación filosófica más amplia sobre la educación humanista que articula el paradigma de la complejidad de Morin con la visión humanista de Lonergan (López-Calva, 2009).

"La reforma del pensamiento conduce a una reforma de vida absolutamente necesaria para el buen vivir..." afirma Morin (2016, p. 102) para subrayar la relación estructural entre pensamiento y ética que en estos tiempos de desafíos interconectados en todos los campos del quehacer humano se traducen en una necesidad de reformar el pensamiento que pueda conducirnos a un cambio de vida que haga realidad la aspiración central de toda ética: el buen vivir o la buena vida humana, que en estos tiempos de globalización se propo- ne entender como una ética planetaria (Morin, 2005). Esta perspectiva supone que la buena vida humana no puede concebirse ni construirse desde una perspectiva meramente individual, porque los seres humanos están intersubjetivamente ligados en un "...nosotros primario que es vital y funcional..." (Lonergan, 1988, p. 61) y que plantea el desafío de construir sociedades humanas estructuradas en torno al bien común, sociedades justas donde sea posible la humanización simultánea de cada persona y del conjunto.

A continuación, se presenta una revisión sintética de estos tres elementos que conforman el bucle en el que se sustenta la propuesta teórica que se indagó de manera exploratoria en la sección del cuestionario que se aplicó a los sujetos de esta investigación: pensamiento complejo-ética planetaria-transformación social.

El pensamiento complejo, afirma Morin (2016) “...es el pensamiento que quiere superar la confusión...y la dificultad de pensar, mediante un pensamiento organizador: un pensamiento que separa y conecta..." (p. 87). Se trata de un pensamiento capaz de mirar siempre las partes y el todo para construir aproximaciones más comprehensivas de los fenómenos y aportar respuestas más integrales e integradoras a los desafíos de la realidad.

Cuando se piensa de una forma compleja, se superan las visiones de causalidad lineal y se asume que en toda realidad vital la causa genera el efecto, pero el efecto regenera la causa, se trasciende la mirada excluyente, que no admite que dos verdades opuestas puedan con frecuencia ser ambas verdaderas, se comprenden los distintos fenómenos de la realidad en las relaciones entre sus partes y de las partes con el todo, asumiendo que el todo está en cada una de las partes, así como las partes están en el todo y se da su lugar al pensamiento lógico y al pensamiento mitológico, como diferentes pero complementarios para un conocimiento que sea realmente humano (Morin, 1999).

Este paradigma propone siete grandes principios que rigen el pensamiento complejo. Se trata del principio sistémico u organizacional, el principio hologramático, el principio dialógico, el principio de retroactividad, el principio de recursividad, el principio de autonomía-dependencia y 
el principio de reintroducción del sujeto cognoscente en su conocimiento (Morin et al., 2006).

Estos principios describen, con mayor precisión y distinción de elementos, las características del pensamiento complejo que se han planteado en los párrafos anteriores. Existe además otro principio que se denomina de ecología de la acción, que está, junto con el de autonomía-dependencia, más directamente relacionado con la dimensión ética del ser humano (Morin, 2005).

Dos de estos principios constitutivos del pensamiento complejo sustentan la relación entre pensar bien y construir bien o contribuir a la edificación del bien humano. El principio de autonomía-dependencia, que plantea que todas las partes de un sistema son relativamente autónomas y capaces de autodeterminarse, pero al mismo tiempo son dependientes de ese sistema con el que necesitan mantenerse unidos para poder sobrevivir, aprender, construir y crecer. Esta noción tiene que ver con la visión de libertad humana en Morin (2003) que es una autonomía siempre sujeta a ciertas dependencias.

El principio de ecología de la acción plantea que cada ser humano es relativamente responsable de sus decisiones y acciones pero que, cuando estas decisiones y acciones entran a jugar dentro del ecosistema de decisiones y acciones de los demás seres humanos o grupos y del papel del azar, pueden tener efectos que escapan del control de quien decidió o actuó en determinado sentido y con ciertas intenciones. Las consecuencias de este rejuego de decisiones y acciones combinadas en un sistema pueden hacer incluso que las decisiones y acciones tengan efectos contrarios e incluso se vuelvan en contra de la persona que las ejecutó. Este principio rompe con la idea individualista de la libertad. Desde la ecología de la acción se ve claramente que la libertad individual está siempre interrelacionada sistémicamente con la libertad de los demás individuos y que toda decisión y acción afecta y es afectada por las de los demás.

El segundo elemento en el bucle de sustento teórico es la ética planetaria, que es una noción compleja que parte de los distintos deberes de religación que experimentan los seres humanos: el deber egocéntrico que lo hace buscar religarse consigo mismo, el deber genocéntrico que lo lle- va a la búsqueda de religación con los suyos, los de su raza o cultura; el deber sociocéntrico que lo mueve al trabajo por la religación con la sociedad y finalmente, el deber antropocéntrico que lo conduce a buscar religarse con la especie humana de la que forma parte.

Estos deberes se traducen en una autoética, una socioética y una antropoética que están interrelacionadas. El punto de partida es la concepción del ser humano como individuo-sociedad-especie de manera simultánea y estructural. La ética planetaria o ética del género humano subraya la relación individuo especie manifiesta en la antropoética, mientras que la socioética subraya la relación individuo-sociedad. La socioética se realiza mediante la regeneración constante de la democracia, mientras que la antropoética se realiza a través de la búsqueda de solidaridad, fraternidad e igualdad entre todos los seres humanos, luchando contra la exclusión para no dejar al margen a ningún ser humano de la humanidad a la que pertenece (Morin, 2005).

Así como es importante la formación del profesorado en el pensamiento complejo, que parece estar ausente por el peso de una tradición formativa hiperespecializada y fragmentada de los saberes, basada en la disyunción, así también la formación docente en una ética planetaria resulta fundamental en estos tiempos de desafíos interconectados, que piden compromiso y solidaridad internacional.

El tercer elemento del bucle que constituye el sustento teórico de este trabajo es el de la transformación social. De la misma forma en que el pensamiento y la ética están estructuralmente ligados, la ética planetaria en su carácter ternario tiene una implicación social inseparable. Esta doble condición plantea por una parte la necesidad, en la relación entre individuo y sociedad, de educación para una ciudadanía democrática en la que los educandos crezcan en la plena conciencia de que se requiere un control mutuo del individuo por la sociedad y de la sociedad por el individuo, para que exista una verdadera democracia.

Por otro lado, se requiere de la formación en la relación individuo-especie que hoy reclama una ciudadanía terrestre, en la que los educandos desarrollen una conciencia de su identidad terrenal y al mismo tiempo que se sienten plenamente 
parte del país en el que han nacido y vivido, se asuman también profundamente como miembros de la Tierra-Patria (Morin, 1993).

La formación ética del docente tiene hoy que responder al cambio de paradigma que incluya la relación entre pensamiento complejo, ética planetaria y transformación social, como ejes de la labor del profesorado.

Esta formación implica una reforma profunda de la concepción del docente como transmisor de contenidos o como enseñante de valores abstractos y modelador del carácter de los estudiantes desde visiones ideales y de perfección, para pasar a la perspectiva que plantea Morin (2016) de director de orquesta que coordina los esfuerzos e imprime un talante a la formación autónoma de cada uno de los educandos, dejando que sean ellos los que busquen la información pero convirtiéndose en un promotor de análisis, síntesis, procesamiento y sobre todo, reflexión crítica y deliberación ética.

Para lograr este cambio paradigmático en la formación de los profesores, un paso central sería el que plantea Morin (2005) para la formación ética de los estudiantes que consiste en lo que él llama trabajar para pensar bien. La educación del paradigma dominante, tanto en el caso de los educandos como en el de los educadores, tiene muchos más elementos de los que Morin (2005) considera como pensar mal: se trata de una formación que enseña a fragmentar los conocimientos en asignaturas y temas separados, que tiende a ignorar los contextos de los conocimientos y solo ve la unidad o la diversidad en los datos, pero no es capaz de ver la unidad en la diversidad y la diversidad en la unidad. Se trata, sobre todo en este contexto actual que Nussbaum (2010) llama Educación para la renta, de una educación que solo ve lo inmediato, ignora el pasado y ve apenas el futuro a corto plazo; una educación que deja de lado la relación recursiva entre pasado-presente-futuro porque mira solo al momento presente. Es una educación que privilegia lo cuantificable y desprecia o minimiza lo que no puede ser medido u observado, por lo que las humanidades y las artes se sacrifican en lugar de magnificarse como debería ser, según lo plantean tanto Morin (2000), como Nussbaum (2010) y Lonergan (1999).

Lo que se requiere en la formación del profe- sorado para habilitarlo como director de orquesta es, por el contrario, educar en un ambiente de trabajo para pensar bien, que según el mismo Morin (2005) consiste en religar, liberar los conocimientos de los fijamientos, abandonar el punto de vista mutilado de las disciplinas y asignaturas separadas y buscar un conocimiento poli, multi e inte o transdisciplinar. Se necesita capacitar al profesorado para lidiar con lo complejo del mundo actual, desarrollar sus capacidades para distinguir e integrar, para separar el reduccionismo del holismo mediante la capacidad de ligar las partes con el todo en cada fenómeno estudiado y en la comprensión del proceso educativo.

Se trata de una formación del profesorado que enseñe a considerar los contextos, que desarrolle una racionalidad abierta y que pueda inscribir las realidades actuales en el círculo pasado-presente-futuro para tener una comprensión integrada del conocimiento y del bien humano en construcción, reconociendo las incertidumbres.

\section{Método}

Como se señaló en la introducción, este artículo presenta los resultados de una primera indagación empírica exploratoria acerca de la propuesta teórica original sobre la formación ética del profesorado sustentada en el bucle que articula dialógicamente tres elementos centrales: pensamiento complejo-ética planetaria-transformación social. En este trabajo se plantean los resultados de una encuesta, en el apartado relacionado con estos tres elementos.

\section{Participantes.}

Se construyó una muestra de oportunidad formada por dos tipos de participantes: por una parte, docentes de distintos niveles educativos y por la otra, académicos expertos en el tema de la formación del profesorado, entre los que se consideró a funcionarios y exfuncionarios del sistema educativo mexicano, directivos de instituciones formadoras de docentes, investigadores educativos en el área de formación del profesorado y formadores de docentes, con el fin de contrastar 
la visión de quienes han vivido los programas de formación y quienes han tenido participación en el diseño, instrumentación o investigación sobre estos programas.

En el caso de los expertos, la encuesta se aplicó a una muestra de oportunidad de 22 académicos de reconocido prestigio en el campo de la formación del profesorado y de la política educativa. La muestra de expertos estuvo conformada por 13 hombres y nueve mujeres, de quienes se presentan algunos datos en la Tabla 1.

La gran mayoría de los encuestados tiene como especialidad la Educación o la Pedagogía (19), aunque varios de ellos tienen además formación en el campo de la Sociología (2), Filosofía (1) y la Tecnología educativa (1).

Sobre sus campos de actividad, la mayoría se dedica prioritariamente a la docencia y la investigación -16 y 15 respectivamente-, 10 han sido o son directivos y cinco más han ocupado cargos como funcionarios públicos, uno de ellos como Secretario de Educación Pública en el sector federal y otro en el ámbito estatal.

En el caso de la muestra de docentes, respondieron el instrumento un total de 222 profesores y profesoras, de los cuales 163 son mujeres y 59 hombres. En sus perfiles de formación predomi- na el grado de maestría con 110 docentes, seguido del de licenciatura con 57 y de doctorado con 55 participantes. A continuación, se presentan algunos datos de estos docentes en la Tabla 2.

\section{Instrumento}

Para este trabajo de investigación se diseñó un instrumento original titulado "La formación de profesores en Iberoamérica: una visión desde la ética profesional". Este cuestionario consta de seis apartados: datos censales, agencia, transformación intelectual, transformación ética - desde la visión de la ética de principios y de la ética planetaria - , pacto educativo y futuro. El total de ítems del instrumento fue de 20 preguntas cerradas estructuradas en una escala Likert de siete opciones que van desde Totalmente en desacuerdo hasta Totalmente de acuerdo y cuatro preguntas abiertas en las últimas dos secciones: pacto educativo y futuro.

El objetivo del cuestionario fue indagar de forma exploratoria la opinión de expertos en el tema de la formación del profesorado y de docentes en ejercicio, acerca de distintos aspectos de la formación ética del profesorado que corresponden a cada apartado de los seis señalados en el párrafo anterior. Como ya se ha expresado, la selección de

\section{Tabla 1}

Datos de los 22 Participantes Expertos

\begin{tabular}{|c|c|}
\hline \multicolumn{2}{|c|}{ Grado académico } \\
\hline Doctorado & $86.4 \%$ \\
\hline Maestría & $13.6 \%$ \\
\hline \multicolumn{2}{|c|}{ Años de experiencia en el campo educativo } \\
\hline Más de 30 años & $77.3 \%$ \\
\hline Entre 30 y 25 & $4.5 \%$ \\
\hline Entre 25 y 21 & $9.1 \%$ \\
\hline Entre 20 y 16 & $9.1 \%$ \\
\hline \multicolumn{2}{|c|}{ Nacionalidad } \\
\hline Mexicanos & $82 \%$ \\
\hline Argentino & $4.5 \%$ \\
\hline Uruguayo & $4.5 \%$ \\
\hline Venezolano & $4.5 \%$ \\
\hline Colombiano & $4.5 \%$ \\
\hline
\end{tabular}


Tabla 2

Datos de los 222 Participantes Docentes

\begin{tabular}{cc}
\hline & Nivel educativo de trabajo \\
\hline Licenciatura & $40.1 \%$ \\
Preescolar & $23.0 \%$ \\
Posgrado & $21.2 \%$ \\
Educación Básica & $19.4 \%$ \\
Bachillerato & $13.5 \%$ \\
Escuelas normales & $13.5 \%$ \\
\hline Sector educativo de trabajo & \\
\hline Público & $55.4 \%$ \\
Privado & $51.8 \%$ \\
\hline Mexicanos & \\
Argentinos & $98.2 \%$ \\
Colombianos & $0.9 \%$ \\
\hline
\end{tabular}

Nota. En los rubros Nivel educativo de trabajo y Sector educativo la suma es mayor al 100\% porque algunos de ellos laboran en varios niveles educativos y en ambos sectores.

ambos grupos tiene que ver con la experiencia desde los ángulos de quien se ha formado en los programas y de quien ha participado en su diseño y operación o en la investigación sobre ellos. El instrumento fue sometido a juicio de expertos por parte de dos académicos que son miembros del Sistema Nacional de Investigadores (SNI) del Consejo Nacional de Ciencia y Tecnología (Conacyt) de México. Ambos investigadores son expertos en temas de metodología de investigación, tienen más de cinco años de experiencia como directores de tesis de maestría y doctorado y como evaluadores de artículos en revistas nacionales e internacionales. Una de ellos es además regularmente, profesora de seminarios de investigación.

El presente artículo reporta los resultados obtenidos de las preguntas relacionadas con el pensamiento complejo, la transformación social y la ética planetaria que corresponden a los números 2.5, 3.4 y 3.11 respectivamente dentro del cuestionario.

\section{Procedimiento}

La invitación a los participantes se realizó utilizando dos estrategias distintas. En el caso de los expertos, se envió a través de correo electrónico y en el de los docentes se hizo de manera pública y abierta.

En el caso de los expertos, se hizo la invitación personalizada a un grupo amplio de expertos alrededor de 35-, la mayor parte conocidos previamente por el investigador y algunos otros que han sido solamente leídos en su producción sobre el tema. El envío de la invitación se realizó de forma personalizada a través del correo electrónico de cada experto, con un texto donde se explicaba sintéticamente el objetivo del cuestionario y se incluía la liga al formato de Google Forms donde podía responderse. La selección de los expertos se circunscribió a la región de Latinoamérica y de este grupo amplio respondieron el cuestionario los 22 señalados.

Para los docentes de distintos niveles educativos, la invitación se realizó abiertamente a través de la página personal de la red social Facebook, en la que se cuenta con una lista de casi 4,000 seguidores, de los cuales un porcentaje aproximado del $60 \%$ son profesores en servicio, estudiantes de licenciaturas y posgrados en educación o investigadores educativos.

La aplicación del instrumento se realizó dando un plazo aproximado de tres semanas para recibir 
las respuestas, en el semestre de otoño de 2020. Una vez terminado el tiempo de recolección se procedió con la obtención de los datos mediante la hoja de Excel que se obtiene automáticamente en Google Forms.

En ambos casos no se pidió la firma de una carta de consentimiento informado puesto que la aplicación del instrumento fue abierta a quienes quisieran responder y el instrumento de Google Forms garantiza el anonimato de quienes responden. Este elemento de confidencialidad de los datos se especificó en el texto de invitación enviado a los expertos por correo electrónico y a los docentes por la página de Facebook.

\section{Análisis de Datos}

Por tratarse como se ha explicado ya de una primera aproximación empírica de carácter exploratorio para conocer la opinión de los expertos y los docentes acerca de la formación ética del profesorado, desde la contribución teórica humanista y compleja sintetizada en este trabajo en el bucle: pensamiento complejo-ética planetaria-transformación social, el análisis de los datos que se presenta en este trabajo es de corte exclusivamente descriptivo.

De esta manera, se presentan los porcentajes de respuestas en cada opción de la escala Likert en las tres preguntas abordadas en el trabajo, tanto en el grupo de expertos como en el de docentes con el fin de comparar y contrastar sus opiniones con respecto a cada uno de los tres conceptos eje de la propuesta teórica. Como parte de este análisis descriptivo se muestra la moda y las tendencias hacia el grado de acuerdo o desacuerdo por parte de ambos grupos de participantes en los tres ítems considerados.

Este análisis descriptivo cumple con el objetivo de indagar de forma exploratoria las opiniones de expertos y docentes sobre la formación ética del profesorado vista desde el bucle pensamiento complejo-ética planetaria-transformación social, aunque evidentemente aporta solamente una primera imagen que necesita ser profundizada mediante el análisis de las preguntas abiertas del cuestionario y para una visión explicativa que trascienda las opiniones y explore los significados y análisis de los participantes de ambos grupos, por una segunda etapa de carácter cualitativo que podría realizarse a través de entrevistas semiestructuradas o de grupos focales por mencionar dos posibles estrategias metodológicas de continuidad de este trabajo.

\section{Resultados}

En este apartado se presentan los resultados de la encuesta exploratoria sobre la formación del profesorado en las preguntas relacionadas con el bucle: pensamiento complejo-ética planetaria-transformación social. En cada una de las figuras se representan en el eje horizontal (x) las siete opciones de respuesta de la escala Likert que se plantearon de la siguiente forma: 1 (totalmente en desacuerdo), 2 (En desacuerdo), 3 (Parcialmente en desacuerdo), 4 (Indiferente), 5 (Parcialmente de acuerdo), 6 (De acuerdo), 7 (Totalmente de acuerdo). En el eje vertical se representan en barras el número de respuestas obtenidas en cada ítem y en la descripción en texto de cada una se plantean los porcentajes que estos números representan con respecto al total de la muestra.

\section{Pensamiento Complejo en la Formación del Profesorado.}

El ítem 2.5 evalúa la opinión sobre el pensamiento complejo. Aunque se muestran aparentemente dos ideas en un solo ítem, se redactó de manera en que el término pensamiento complejo fuese desglosado en términos más concretos como pensamiento que integra las diversas disciplinas que constituyen la formación en lugar de separarlas y aislarlas.

Como puede apreciarse en la figura 1, las respuestas de los docentes se orientan más hacia la opinión de que los programas de formación del profesorado desarrollan moderadamente el pensamiento complejo y una visión integradora de las distintas disciplinas que explican el fenómeno educativo. La moda de las respuestas se encuentra en la respuesta Parcialmente de acuerdo en el que se ubica el $31.5 \%$ de los profesores que 
respondieron y si se suman las respuestas de esta opción con las de la respuesta De acuerdo, encontramos casi la mitad de los profesores encuestados (49.5\%). La curtosis de la curva tiene una tendencia moderada hacia el lado positivo de las respuestas, lo que parece indicar que, si bien un buen porcentaje de los profesores considera que se atiende el rubro del pensamiento complejo y su visión integradora, no tienen una percepción contundente de que se atienda este eje formativo.
Por su parte, la figura 2 muestra una curtosis más orientada hacia el lado negativo de la curva, con una moda en la respuesta 2: En desacuerdo que tiene el $27.3 \%$ de las respuestas - casi una tercera parte- - y si se suma a las opciones de Totalmente en desacuerdo y Parcialmente en desacuerdo se concentra el 59.1\%, lo cual indica que los exfuncionarios públicos -exsecretarios de Educación Pública estatal y uno federal-, exdirectivos o formadores de docentes e investigadores educativos especializados en el tema de la

\section{Figura 1}

Pensamiento Complejo (Visión de los Docentes)

2.5 La formación docente promueve el pensamiento complejo y la integración de las disntistas disciplinas que explican el fenómeno educativo. 222\&nbsp;respuestas

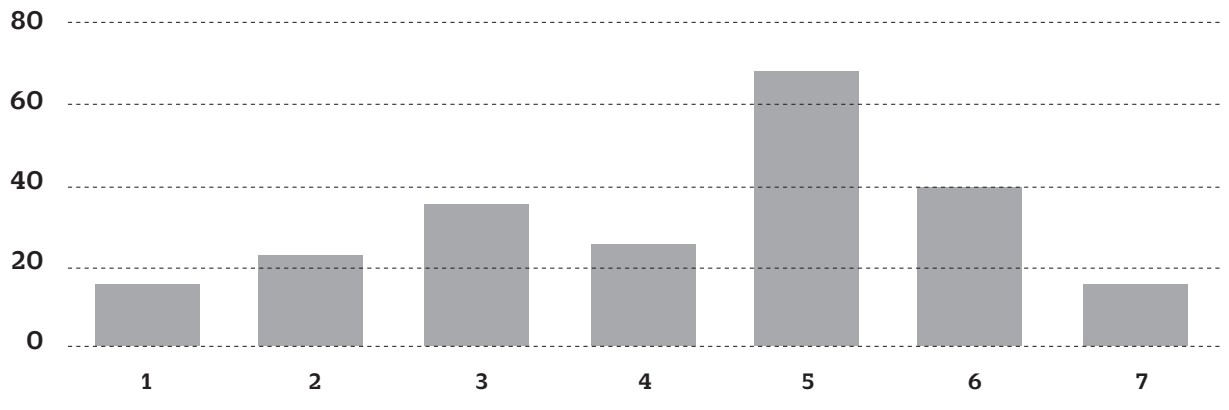

\section{Figura 2}

Pensamiento Complejo (Visión de los Expertos)

2.5 La formación docente promueve el pensamiento complejo y la integración de las disntistas disciplinas que explican el fenómeno educativo. 222\&nbsp;respuestas

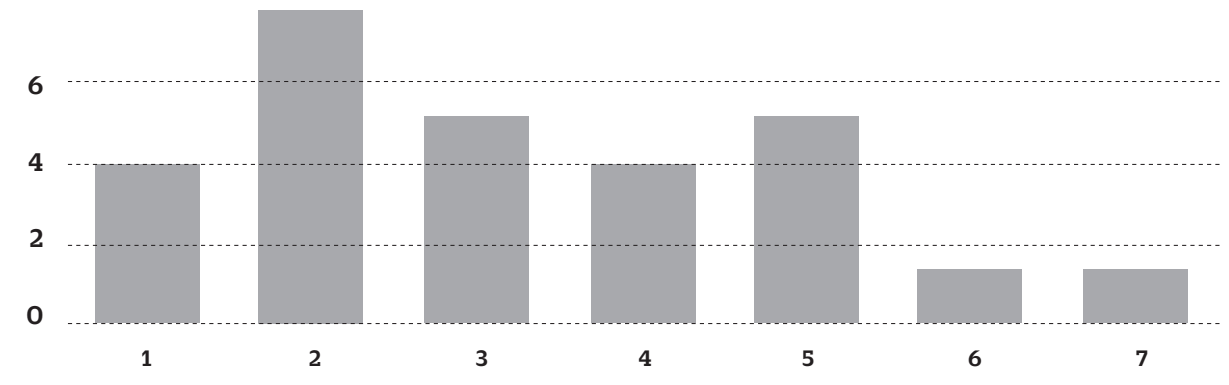


formación docente, tienden a pensar que existe una carencia en la formación de pensamiento complejo y de una visión integradora de las disciplinas dentro de los programas de formación del profesorado en América Latina.

\section{Transformación Social}

La pregunta que indagó la opinión de ambos grupos de participantes sobre la transformación social es la 3.4 del cuestionario, que está formulada así: Los programas de formación docente desarrollan una conciencia operante sobre el compromiso ético del profesor en la transformación social.

En este componente de transformación social en la formación del profesorado, entendido como un compromiso ético de todo educador, las tendencias vuelven a ser encontradas, con una visión moderadamente positiva de los profesores (figura 3) - un poco más alta que en el rubro de pensamiento complejo- mientras los expertos (figura 4) vuelven a mostrar una visión más crítica de los programas con respecto al compromiso ético orientado hacia la transformación social desde la docencia.

En el caso de los profesores, curtosis de la curva es hacia el lado positivo, teniendo la moda en la respuesta Parcialmente de acuerdo, con prácti- camente la cuarta parte de las respuestas (24.8\%) y si se suman las respuestas que van de Parcialmente de acuerdo a Totalmente de acuerdo pasando por la respuesta De acuerdo, se encuentran ahí el 53.9\% de las respuestas obtenidas.

En las respuestas proporcionadas por los expertos, la curva tiene una curtosis hacia el lado negativo sin tener respuestas en el punto 1 de la escala que ubicaba la respuesta Totalmente en desacuerdo. La moda se encuentra en la respuesta Parcialmente en desacuerdo con un 31.8\% y si se suman esta respuesta y las de En desacuerdo e Indiferente, se obtiene un $77.2 \%$ del total de las respuestas. Como se puede inferir, aunque la respuesta negativa también es un poco más moderada que en el rubro de pensamiento complejo, los expertos consideran en general que es insuficiente la formación del compromiso ético del profesor orientado hacia la transformación social a través de su labor educativa.

Si consideramos que la relación entre compromiso ético y transformación social a nivel nacional y planetario es fundamental desde la visión de complejidad que asume esta investigación como fundamento necesario para una formación docente que responda a los desafíos profundos e interconectados que caracterizan al mundo en esta crisis

Figura 3

Transformación Social (Visión de los Expertos)

3.4 Los programas de formación docente desarrollan una conciencia operante sobre el compromiso ético del profesor en la transformación social. 22\&nbsp;respuestas

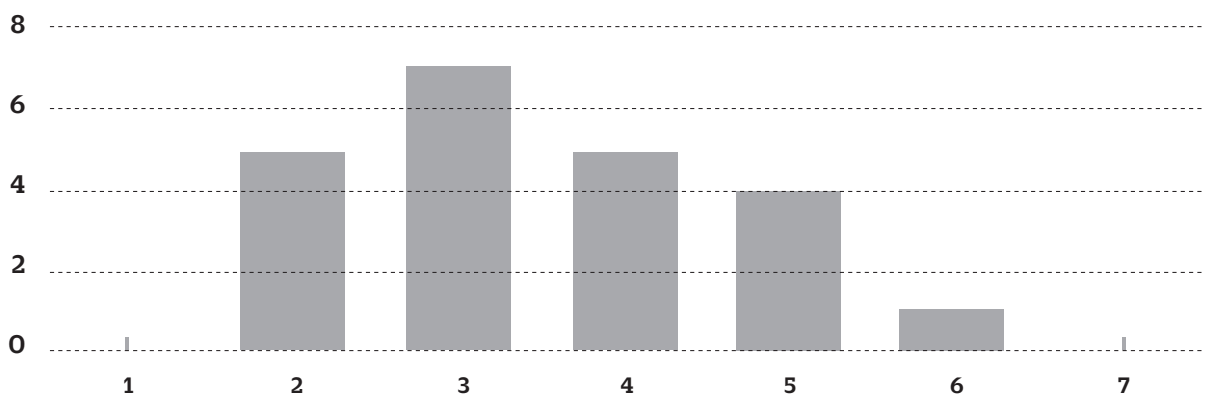




\section{Figura 4}

Transformación Social (Visión de los Docentes)

3.4 Los programas de formación docente desarrollan una conciencia operante sobre el compromiso ético del profesor en la transformación social.

22\&nbsp;respuestas

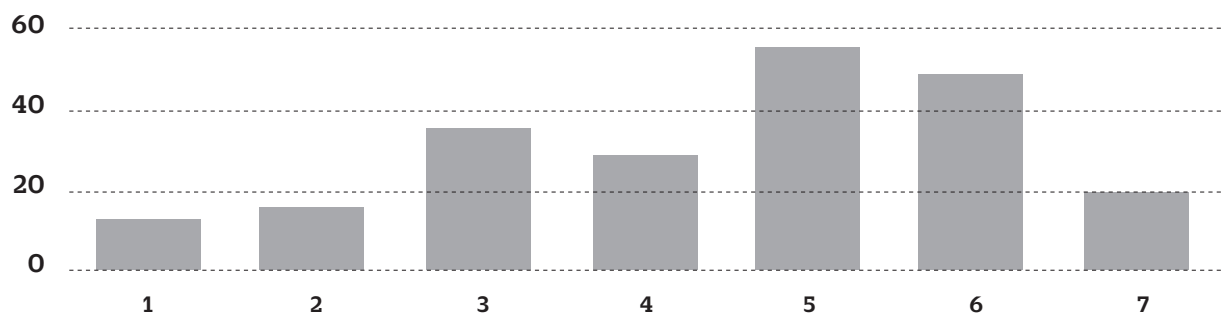

civilizatoria, puede verse que aunque los docentes tienen una mayor inclinación a pensar en que en la formación del profesorado se está trabajando este componente, esta tendencia no es suficiente, sobre todo considerando la evidente contradicción con lo que plantean los expertos en el tema.

\section{Ética Planetaria}

El ítem orientado a la obtención de la opinión de ambos grupos de participantes sobre la formación docente en una visión de ética planetaria es la 3.11 del cuestionario que se formuló de la siguiente manera: La formación de profesores desarrolla la conciencia planetaria y el sentido de compromiso con la humanidad como noción ética. De la misma forma que en el caso del pensamiento complejo, la redacción aparentemente contiene dos ideas distintas, sin embargo, la segunda parte del ítem es solamente una explicitación de la primera, es decir, una conciencia planetaria consiste en un compromiso con la humanidad como noción ética, tal como lo explica Morin (2001).

En este tercer componente del bucle pensamiento complejo-transformación social-ética planetaria, encontramos nuevamente el mismo patrón en el que los docentes en general se orientan, aunque de manera no contundente, hacia una visión más positiva de la formación del profesora- do, mientras que los expertos vuelven a mostrar una tendencia más crítica.

La figura 5, que presenta las respuestas de los profesores tiene una curtosis claramente orientada hacia el lado positivo de la curva, aunque las respuestas están bastante distribuidas entre las diversas opciones de la escala. La moda de esta gráfica se encuentra dividida en partes prácticamente iguales entre las opciones de Parcialmente de acuerdo (23.4\%) y De acuerdo (23.9\%) lo que ubica una tendencia moderadamente positiva del $47.3 \%$ que sumada con la opción De acuerdo total (6.3\%) representa el 53.6\%, es decir, prácticamente la mitad de los profesores encuestados. Sin embargo, no hay que dejar de considerar que prácticamente un tercio de los docentes que respondieron el instrumento (33.3\%) lo hicieron En desacuerdo, Desacuerdo parcial o total.

En la figura 6 que muestra los resultados de las respuestas de los expertos, se encuentra una tendencia claramente negativa con respecto a que los programas de formación del profesorado estén trabajando en el desarrollo de una conciencia planetaria y en el sentido de compromiso con la humanidad como noción ética, según plantea Morin (2001). La moda se encuentra en la respuesta de En desacuerdo con $36.4 \%$ de las respuestas que, sumadas a las de Desacuerdo total, represen- 
tan el 59.1\% de las respuestas, cercana a las dos terceras partes de los sujetos encuestados.

Por su parte, las respuestas del lado positivo de la gráfica se ubican en la opción Parcialmente de acuerdo con el $18.2 \%$ de las respuestas y solamente hubo una respuesta que mostró un Acuerdo total con la formulación de este ítem.

Estos resultados evidencian que los expertos consideran en su mayoría que este rubro de la ética planetaria es el que está más ausente en los programas de formación del profesorado en la región latinoamericana, cuestión que si bien re- sulta comprensible, porque la ética planetaria o del género humano es un concepto relativamente reciente en el pensamiento filosófico - aunque data de finales del siglo pasado- es preocupante debido a la urgencia que tiene el mundo fragmentado y excluyente en el que hoy se vive, de una formación ética con visión incluyente y centrada en la formación de una ciudadanía terrestre y en una consciencia de que los humanos se definen estructuralmente desde la triunidad individuo-sociedad-especie, según plantea Morin en su visión antropológica (2003).

\section{Figura 5}

Ética Planetaria (Visión de los Docentes)

2.5 La formación docente promueve el pensamiento complejo y la integración de las disntistas disciplinas que explican el fenómeno educativo. 222\&nbsp;respuestas

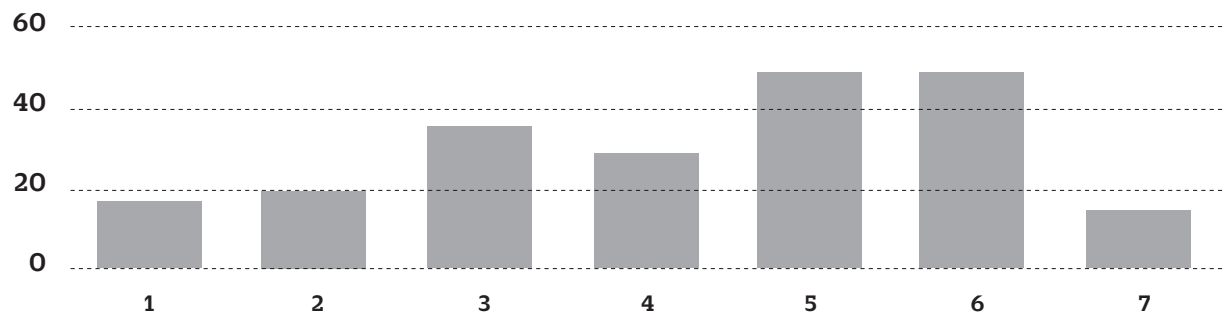

\section{Figura 6}

Ética Planetaria (Visión de los Expertos)

2.5 La formación docente promueve el pensamiento complejo y la integración de las disntistas disciplinas que explican el fenómeno educativo. 222\&nbsp;respuestas

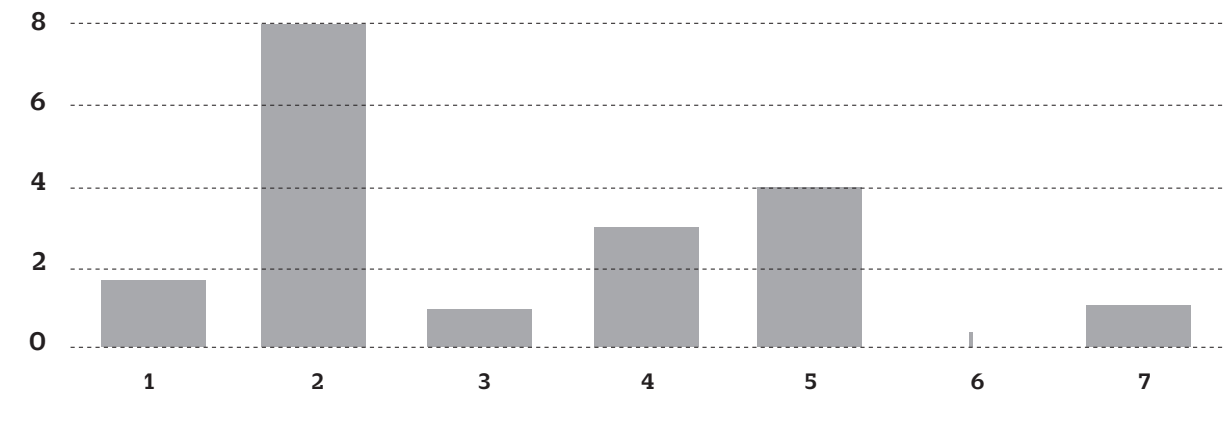




\section{Discusión}

El mundo vive una crisis muy profunda que presenta desafíos interconectados de carácter ambiental, económico, social, político, cultural que están poniendo en una verdadera encrucijada a las sociedades nacionales y a las estructuras globales de mercado, que no han logrado ser compensadas por el desarrollo de una política de humanidad que fomente la responsabilidad y la solidaridad planetaria. Se trata de una crisis sistémica que muchos autores han nombrado de formas diferentes, pero que es en el fondo una crisis de humanidad que requiere de la ética para poder ser resuelta.

Desde el paradigma de la complejidad, esta necesidad de desarrollo de la ética para enfrentar los graves problemas de este cambio de época no está separado sino estructuralmente articulado con el desarrollo del pensamiento: la transformación moral y la transformación del pensamiento están unidas de forma inseparable formando un bucle recursivo y retroactivo.

Una condición necesaria para desarrollar esta reforma es la formación de los profesores, que son los sujetos centrales en la educación de los futuros ciudadanos. La contribución central de este artículo consiste en afirmar que esta formación tiene que sustentarse en el bucle pensamiento complejo-ética planetaria-transformación social y presentar una primera aproximación empírica sobre esta perspectiva.

Para explorar la opinión de docentes de distintos niveles educativos y de expertos en formación del profesorado sobre hasta qué punto está o no presente este bucle en los programas de formación del profesorado, se han expuesto en este artículo los resultados de una indagación de carácter exploratorio, aplicada a una muestra de ambos grupos.

Los resultados muestran que, aunque los docentes tienen una opinión más positiva que los expertos, el bucle pensamiento complejo-ética planetaria-transformación social no está suficientemente presente en los programas de formación del profesorado, según la opinión de ambos grupos.

Por tratarse de una primera aproximación empírica y ser un estudio de carácter exploratorio y exclusivamente descriptivo, estos resultados tienen limitaciones y deberán ser complementados con una etapa posterior de investigación de carácter cualitativo en la que se indaguen, más allá de la opinión, los significados y los elementos teóricos y experienciales que sustentan la visión de los docentes y de los expertos en la formación del profesorado en el tema de la formación ética desde esta mirada humanista y compleja. Ante la crisis de humanidad que vive el mundo de hoy, resulta urgente enfatizar la formación ética del profesorado.

\section{Referencias}

Belavi, G., \& Murillo, F.J. (2020). Democracia y Justicia Social en las Escuelas: Dimensiones para Pensar y Mejorar la Práctica Educativa. REICE. Revista Iberoamericana sobre Calidad, Eficacia y Cambio en Educación, 2020, 18(3), 5-28. https://doi.org/10.15366/reice2020.18.3.001

Gore, A. (2006). An inconvenient truth: the planetary emergency of global warming and what can we do about it. Rodale Books.

Gorostiaga, X. (2000). En busca del eslabón perdido entre educación y desarrollo. Revista Latinoamericana de Estudios Educativos, 30(1), 11-66. https://www. redalyc.org/pdf/270/27030102.pdf

Hirsch, A. (2009) Principios y reglas de la ética profesional para el proyecto interuniversitario sobre ética profesional en México. En J.A. Ibáñez-Martín (Coord.), Educación, Conocimiento y Justicia (pp. 321 -331). Dykinson.

Hirsch, A. (2013). La ética profesional basada en principios y su relación con la docencia. Edetania 43, 98-111. https://dialnet.unirioja.es/ejemplar/342477

Hirsch, A. (2021). La contribución de la universidad para mejorar el trabajo académico de los profesores de posgrado. Archivos Analíticos de Políticas Educativas, 29(9). https://doi.org/10.14507/epaa.29.5594

Jover, G., \& Ruiz, M. (2013). El código deontológico de la profesión docente. Evolución y posibilidades. Edetania 43, 113-131. Consultado en: https://dialnet.unirioja. es/ejemplar/342477

Lonergan, B. (1988a). Método en Teología. Sígueme.

Lonergan, B. (1998b). Filosofía de la Educación. Universidad Iberoamericana.

Lonergan, B. (1999). Insight. Estudio sobre la comprensión humana. Sígueme-Universidad Iberoamericana.

López-Calva, M. (2009). Educación humanista. Tres volúmenes. Gernika.

Meirieu, P. (2022). El futuro de la Pedagogía. Teoría de la 
Educación. Revista Interuniversitaria, 34(1), 69-81. https://doi.org/10.14201/teri.27128

Morin, E. (1993). Tierra-Patria. Kairós.

Morin, E. (1999). El Método III. El conocimiento del conocimiento. Ediciones Cátedra.

Morin, E. (2000). La mente bien ordenada. Seix Barral.

Morin, E. (2001). Los siete saberes necesarios para la educación del futuro. UNESCO.

Morin, E. (2003). El Método V. La humanidad de la humanidad. La identidad humana. Ediciones Cátedra.

Morin, E. (2005). O Método VI. Ética. Sulina.

Morin, E. (2016). Enseñar a vivir. Manifiesto para cambiar la educación. Paidós.

Morin, E. (2020). Cambiemos de vía. Lecciones de la pandemia. Paidós.

Morin, E., Roger, E., \& Motta, R. (2006). Educar en la era planetaria. GEDISA. Primera reimpresión.

Nussbaum, M. (2010). Sin fines de lucro. Por qué la democracia necesita de las Humanidades. Katz.
Organización de las Naciones Unidas (2020). Informe de los Objetivos de Desarrollo Sostenible 2020. ONU. https://unstats.un.org/sdgs/report/2020/The-Sustainable-Development-Goals-Report-2020_Spanish. pdf

Pérez-Castro, J., \& Piña, J.M. (2021). La ética profesional de profesores de educación media superior. La visión estudiantil. Diálogos sobre Educación, 23(12), 2007. 2171. La ética profesional de profesores de educación media superior. La visión estudiantil | Diálogos sobre educación (udg.mx)

Ramos, G., \& López, A. (2019). Formación ética profesional y ética profesional del docente. Estudios Pedagógi$\cos \mathrm{XLV}, 3$, 185-199, 2019. https://doi.org/10.4067/ S0718-07052019000300185

Touraine, A. (1994). Crítica de la modernidad. FCE.

Wallerstein, I. (2007). La crisis estructural del capitalismo. Ediciones desde abajo.

RIDU / Revista Digital de Investigación en Docencia Universitaria / e-ISSN: 2223-2516

(C) Los autores. Este artículo es publicado por la Revista Digital de Investigación en Docencia Universitaria del Área de Institutional Research and Effectiveness de la Dirección de Aseguramiento de la Calidad, Universidad Peruana de Ciencias Aplicadas. . "Este es un artículo de acceso abierto, distribuido bajo los términos de la LicenciaCreativeCommons Atribución 4.0 Internacional (CC BY 4.0) (https://creativecommons.org/licenses/by/4.0/deed.es), que permite el uso, distribución y reproducción en cualquier medio, siempre que la obra original sea debidamente citada." 